\title{
Single and Double Charge Exchange Excitations of Spin-Isospin Mode
}

\author{
Hiroyuki Sagawa ${ }^{1,2, *}$ \\ ${ }^{1}$ RIKEN Nishina Center, Wako, Saitama 351-0198, Japan \\ ${ }^{2}$ Center for Mathematics and Physics, University of Aizu, Aizu-Wakamatsu, Fukushima 965-8580, Japan
}

\begin{abstract}
We study the sum rules of double Gamow-Teller (DGT) excitations through double spin-isospin operator $\left(\sigma t_{-}\right)^{2}$. In general, $2^{+}$states in the granddaughter nuclei have dominant transition strength in DGT excitations and $0^{+}$states are weak, except in $T=1$ mother nuclei in which $0^{+}$strength is competitive with $2^{+}$strength. A possibility to extract the unit cross section for the DGT transition strength is pointed out in the $\left(\sigma t_{-}\right)^{2}$ excitation of double isobaric analog state (DIAS) in $T=1$ nuclei.
\end{abstract}

\section{Introduction}

The possibility to induce double charge-exchange (DCX) excitations by means of heavy-ion beams at intermediate energies $[1,2]$ has recently fostered the interest on new collective excitations such as double isobaric analog states (DIAS) and double Gamow-Teller giant resonances (DGTR). In the 1980s, DCX reactions were performed by using pion beams, i.e., $\left(\pi^{+}, \pi^{-}\right)$and $\left(\pi^{-}, \pi^{+}\right)$reactions have been studied. Through these experimental investigations, the DIAS, the dipole giant resonance built on the isobaric analog state (IAS) and the double dipole resonance states were identified [3-6]. However, the DGTRs were not found in the pion double charge-exchange spectra. In the middle of the 1990s, the heavy-ion DCX experiments were performed at energies of 76 and $100 \mathrm{MeV} / \mathrm{u}$, with the hope that the DGTR might be observed in the ${ }^{24} \mathrm{Mg}\left({ }^{18} \mathrm{O},{ }^{18} \mathrm{Ne}\right){ }^{24} \mathrm{Ne}$ reaction [7]. However, no clear evidence of DGTR was found in this reaction. This is mainly because the $\left({ }^{18} \mathrm{O},{ }^{18} \mathrm{Ne}\right)$ reaction is a $(2 n, 2 p)$ type reaction, and even the single GTR in the $t_{+}$channel induced by the $(n, p)$ reaction is weak in $N=Z$ nuclei such as ${ }^{24} \mathrm{Mg}$. A research program based on a new reaction, namely $\left({ }^{12} \mathrm{C}\right.$, ${ }^{12} \mathrm{Be}\left(0_{2}^{+}\right)$) has been planned at the RIKEN RIBF facility with high intensity heavy-ion beams at the optimal energy of $\mathrm{E}_{\mathrm{lab}}=250 \mathrm{MeV} /$ nucleon to excite the spin-isospin response [8]. A big advantage of this reaction is based on the fact that it is a $(2 p, 2 n)$ type DCX reaction and one can use a neutron-rich target to excite DGTR strength. Although many theoretical efforts have been devoted to studies of double $\beta$-decays, DGTR strengths corresponding to the double $\beta$-decays are still too small to be identified in these experiments. Recently, shell-model calculations were performed to study the DGTR of ${ }^{48} \mathrm{Ti}$ [9], and also Ti-isotopes [10]. At the same time, DGTR strength distributions in several nuclei have been studied by using the sum rule approach [11-15]. In ref.[14], the DGT and DIAS states in

\footnotetext{
*e-mail: sagawa@ribf.riken.jp
}

$T=1$ nuclei were studied in the same framework in order to establish a possible unit cross section of DGTR in comparison with the DIAS. The sum rule approach is further extended in ref. [15] to evaluate the energy difference between DGT and DIAS by using separable-type interactions. Minimally-biased theoretical predictions based on sum rules will provide a robust and global view of the DGTR, and can provide a good guideline for the future experimental studies.

\section{Sum Rules for Double Spin-Isospin Phonon States}

The sum rule for the single GT transitions is well known and proportional to the neutron excess,

$$
\begin{aligned}
S_{-}-S_{+}= & \sum_{f}\left|\left\langle f\left|\hat{O}_{-}(\mathrm{GT})\right| i\right\rangle\right|^{2}-\sum_{f}\left|\left\langle f\left|\hat{O}_{+}(\mathrm{GT})\right| i\right\rangle\right|^{2} \\
& =3(N-Z)
\end{aligned}
$$

where the GT transition operators read

$$
\hat{O}_{ \pm}(\mathrm{GT})=\sum_{\alpha} \sigma(\alpha) \mathbf{t}_{ \pm}(\alpha)
$$

The GT sum rule is model independent and gives a good guidance to perform the single charge exchange reactions such as $(\mathrm{p}, \mathrm{n})$ and $\left({ }^{3} \mathrm{He}, \mathrm{t}\right)$ reactions, to observe GTR strength in many nuclei (see for example a review article ref. [16]). Calculated results of single charge exchange GT strength for $t_{-}$and $t_{+}$channels are shown in Fig. 2. A self-consistent Hartree-Fock (HF) + random phase approximation (RPA) are applied with Skyrme EDF SAMi [17] and SAMi-T [18] without and with tensor interactions, respectively. Two peak positions of $t_{-}$channels are reasonably well described by RPA calculations. The peak for $t_{+}$channel is also reproduced well by the RPA calculations. For both channels, however, the absolute magnitudes are over-estimated, i.e., by $35 \%$ for $t_{-}$channel and 


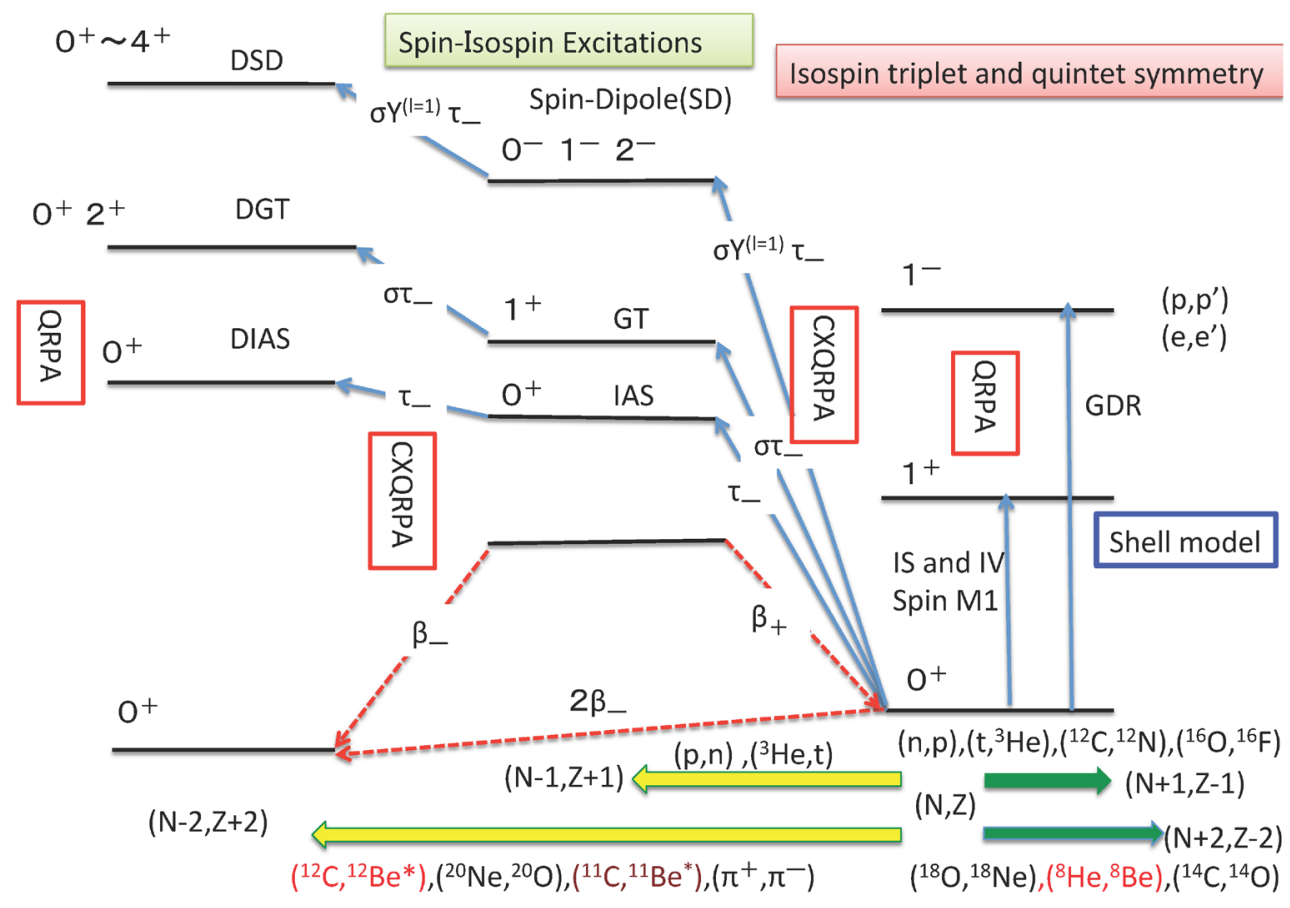

Figure 1. Single- and double-charge exchange excitations and their possible experimental probes. Spin-isospin mode is excited by various charge exchange reactions. $(p, n)$ and $\left({ }^{3} \mathrm{He}, t\right)$ are well adopted for single-charge exchange reactions in experimental studies. Heavy Ion charge exchange reactions are also available to perform double charge exchange reactions. From theoretical sides, RPA, Quasi-particle RPA (QRPA) and charge exchange QRPA (CXQRPA) are often used to study these excitations and modern large scale shell model calculations are also available for the theoretical study.

more for $t_{+}$channel. The quenching factor $q f=0.64$ is multiplied on the GT strength of $t_{-}$channel. These calculations certainly give a confidence for the application of $\mathrm{HF}+\mathrm{RPA}$ model for double charge exchange excitations.

The DGT transition operator $\hat{O}_{ \pm}(\mathrm{GT})^{2}$ can be projected to good multipole states to be

$$
\left[\hat{O}_{ \pm}(\mathrm{GT}) \times \hat{O}_{ \pm}(\mathrm{GT})\right]^{(J)}, J=0,2
$$

The sum rule strength is given by the reduced matrix element,

$$
D_{ \pm}^{(J)}=\frac{1}{2 J_{i}+1} \sum_{f}\left|\left\langle f\left\|\left[\hat{O}_{ \pm}(\mathrm{GT}) \times \hat{O}_{ \pm}(\mathrm{GT})\right]^{(J)}\right\| i\right\rangle\right|^{2}
$$

where $J_{i}$ is the angular momentum of the initial state and the double bars mean the reduced matrix element for the angular momentum. The sum rule values are evaluated by using the double commutator as

$$
\begin{aligned}
D_{-}^{(J=0)}- & D_{+}^{(J=0)}=\left\langle i\left|\left[\left[\hat{O}_{+} \times \hat{O}_{+}\right]^{(J=0)},\left[\hat{O}_{-} \times \hat{O}_{-}\right]^{(J=0)}\right]\right| i\right\rangle \\
=2(N-Z)(N-Z+1) & \\
& +\frac{4}{3}\left[(N-Z) S_{+}-\left\langle i\left|\left[i \hat{\Sigma} \cdot\left(\hat{O}_{-} \times \hat{O}_{+}\right)+\hat{\Sigma} \cdot \hat{\Sigma}\right]\right| i\right\rangle\right]
\end{aligned}
$$

where

$$
\hat{\Sigma}=\sum_{\alpha} \sigma(\alpha)
$$

and $\left(\hat{O}_{-} \times \hat{O}_{+}\right)$is the vector product of two operators. In the last line of Eq. (5), we use an identity

$$
\left(\hat{O}_{+} \times \hat{O}_{-}\right) \cdot \hat{\Sigma}=-\hat{\Sigma} \cdot\left(\hat{O}_{-} \times \hat{O}_{+}\right)+2 i \hat{\Sigma} \cdot \hat{\Sigma}
$$

which makes the final formula of the sum rule (5) different from those in the references $[11,13]$, but it is equivalent to the one in ref. [12]. It is important for quantitative study to rewrite the formula by using the identity (7) since the terms including $\hat{O}_{+}$on the right can be omitted in $N>Z$ nuclei in a good accuracy. The sum rule for $J^{\pi}=2^{+}$final states can be obtained in a similar way to that for $J=0$ states;

$$
\begin{aligned}
& D_{-}^{(J=2)}-D_{+}^{(J=2)} \\
& \quad=\left\langle i\left|\sum_{\mu}(-1)^{\mu}\left[\left[\hat{O}_{+} \times \hat{O}_{+}\right]_{\mu}^{(J=2)},\left[\hat{O}_{-} \times \hat{O}_{-}\right]_{-\mu}^{(J=2}\right]\right| i\right\rangle \\
& =10(N-Z)(N-Z-2) \\
& +\frac{10}{3}\left[2(N-Z) S_{+}+\left\langle i\left|\left[i \hat{\Sigma} \cdot\left(\hat{O}_{-} \times \hat{O}_{+}\right)+\hat{\Sigma} \cdot \hat{\Sigma}\right]\right| i\right\rangle\right]
\end{aligned}
$$

It should be noticed that the sum rule for $J=2$ final states are not given in refs. [11, 12], but given in ref. [13] in a different form.

Next, we consider the DGT transition to the DIAS state. The DIAS state is defined as

$$
|\mathrm{DIAS}\rangle=\frac{T_{-} T_{-}}{N_{f}}|i\rangle
$$



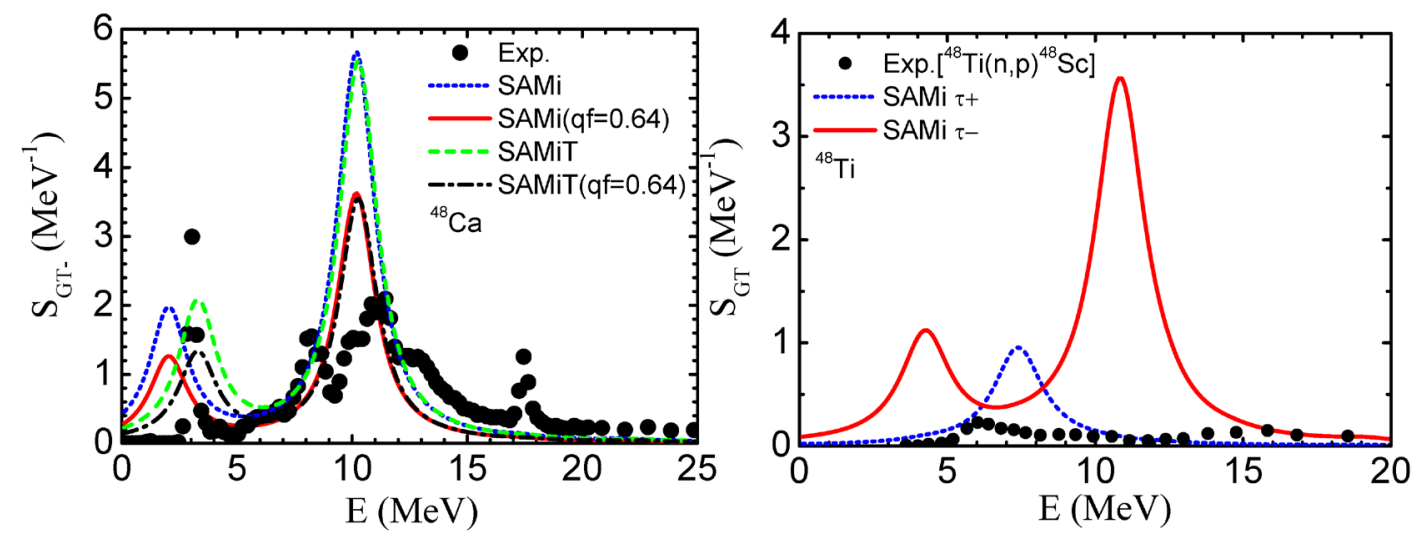

Figure 2. Calculated results of single charge exchange GT strength for $t_{-}$and $t_{+}$channels. Skyrme EDF SAMi and SAMi+T are used for self-consistent HF+RPA calculations. For $t_{-}$channel, the quenching factor $q f=0.64$ are introduced for GT transition strength. Experimental data are taken from ref. [19]. Courtesy of L.G Cao.

Table 1. Various sum rule values for DGT transitions to $J^{\pi}=0^{+}, 2^{+}$and DIAS states given in Eqs. (5), (8) and (11), respectively. The initial state is listed in the first column with the isospin $T$. The double Fermi transitions to DIAS (10) are listed in the fourth column.

For Eqs. (5) and (8), the spin sum rule $\hat{\Sigma} \cdot \hat{\Sigma}$ terms in the last line is obtained by shell model calculations except ${ }^{90} \mathrm{Zr}$. The single particle value of $\Sigma_{\text {sum }}$ is used for ${ }^{90} \mathrm{Zr}$. The single particle values from Eq. (13) are given in the bracket in the sixth column. In the second and third columns, the value in the bracket is the upper limit for $J=0$, while that is the lower limit for $J=2$. The values $\Sigma_{\text {sum }}$ for DIAS ${ }^{(J=0)}(D G T)$ in Eq. (11) are calculated by using the same shell model calculations as for the DGT sum rules, The value $\operatorname{DIAS}^{(J=0)}(\mathrm{DGT})$ with the single particle value of $\Sigma_{\text {sum }}$ in Eq. (13) is also listed in the fifth column with brackets. See the text for more details.

\begin{tabular}{cccccc}
\hline Initial state $(T)$ & $(J=0)$ & $(J=2)$ & DIAS & DIAS(DGT) & $\Sigma_{\text {sum }}$ \\
\hline${ }^{6} \mathrm{He}(1)$ & $12.0(12)$ & $0.0(0)$ & 4 & $3.70(3.70)$ & $0.211 \times 10^{-3}(0.667)$ \\
${ }^{8} \mathrm{He}(2)$ & $39.7(40)$ & $80.7(80)$ & 24 & $7.71(2.47)$ & $0.052(1.333)$ \\
${ }^{14} \mathrm{C}(1)$ & $8.98(12)$ & $7.55(0)$ & 4 & $4.56(0.15)$ & $0.566(1.333)$ \\
${ }^{18} \mathrm{O}(1)$ & $10.4(12)$ & $3.96(0)$ & 4 & $7.72(2.61)$ & $0.297(0.80)$ \\
${ }^{20} \mathrm{O}(2)$ & $35.5(40)$ & $91.3(80)$ & 24 & $4.13(1.74)$ & $0.845(1.60)$ \\
${ }^{42} \mathrm{Ca}(1)$ & $8.50(12)$ & $8.75(0)$ & 4 & $3.80(2.18)$ & $0.66(0.86)$ \\
${ }^{44} \mathrm{Ca}(2)$ & $32.6(40)$ & $98.5(80)$ & 24 & $2.31(1.47)$ & $1.38(1.71)$ \\
${ }^{46} \mathrm{Ca}(3)$ & $72.3(84)$ & $269.3(240)$ & 60 & $1.89(1.32)$ & $2.20(2.57)$ \\
${ }^{48} \mathrm{Ca}(4)$ & $135.5(144)$ & $501.2(480)$ & 112 & $3.70(1.25)$ & $1.59(3.43)$ \\
${ }^{90} \mathrm{Zr}(5)$ & $196.3(220)$ & $859.2(800)$ & 180 & $(1.11)$ & $(4.44)$ \\
\hline
\end{tabular}

where $T_{-}=\sum_{\alpha} t_{-}(\alpha)$ is the isospin lowering operator and the normalization factor is $N_{f}=\sqrt{2(N-Z)(N-Z-1)}$. The double Fermi transition to DIAS state is evaluated to be

$$
\left|\left\langle\mathrm{DIAS}\left|T_{-} T_{-}\right| i\right\rangle\right|^{2}=2(N-Z)(N-Z-1)
$$

in which the mother state is assumed to have a good isospin quantum number $T=T_{z}=(N-Z) / 2$. The DGT transition to DIAS is also expressed as

$$
\begin{aligned}
& D_{-}^{(J=0)}(\mathrm{DIAS})=\left|\left\langle\mathrm{DIAS}\left|\left[\hat{O}_{-} \times \hat{O}_{-}\right]^{(J=0)}\right| i\right\rangle\right|^{2} \\
= & \left|\frac{1}{N_{f}}\left\langle\hat{0}\left|T_{+} T_{+}\left[\hat{O}_{-} \times \hat{O}_{-}\right]^{(J=0)}\right| i\right\rangle\right|^{2} \\
= & \frac{1}{3 N_{f}^{2}}\left[8 \Sigma_{\text {sum }}-2\left(S_{-}+S_{+}\right)\right]^{2} \\
= & \frac{1}{6(N-Z)(N-Z-1)}\left[8 \Sigma_{\text {sum }}-2\left(S_{-}+S_{+}\right)\right]^{2}
\end{aligned}
$$

where $\Sigma_{\text {sum }}$ is the sum rule of the isovector spin transition,

$$
\Sigma_{\text {sum }}=\left\langle i\left|\sum_{\alpha, \mu} \sigma_{\mu}(\alpha) t_{z}(\alpha) \sum_{\beta, \mu^{\prime}} \sigma_{\mu^{\prime}}(\beta) t_{z}(\beta)\right| i\right\rangle .
$$

For a single-j configuration with the occupation probability $v^{2}$, the value $\Sigma_{\text {sum }}$ is evaluated to be

$$
\begin{aligned}
\Sigma_{\text {sum }} & =\sum_{p, h, \mu}\left|\left\langle(p h) 1 \mu\left|\sigma_{\mu} t_{z}\right| \hat{0}\right\rangle\right|^{2} \\
& =\frac{v_{h}^{2}}{4} \frac{\left(2 j_{h}+1\right)\left(2 j_{h}-1\right)}{j_{h}} \text { for } j_{h}=j_{p}+1
\end{aligned}
$$

where we use a filling approximation for the last occupied orbit.

Calculated sum rule values for DGT transitions to $J^{\pi}=$ $0^{+}$and $2^{+}$in Eqs. (5) and (8), respectively, are listed in Table 1 for 10 nuclei, together with those for DIAS states by Fermi and GT operators (10) and (11). In $N>Z$ nuclei with the good isospin quantum number, the vector product 
$\left(\hat{O}_{-} \times \hat{O}_{+}\right)$and the sum $S_{+}$in Eqs. (5) and (8) vanish because of the isospin raising operator in $\hat{O}_{+}$. The spin sum rule $\hat{\Sigma} \cdot \hat{\Sigma}$ term is obtained by shell model calculations. The single particle value is used for ${ }^{90} \mathrm{Zr}$ assuming the neutron excitation from $1 g_{9 / 2}$ to $1 g_{7 / 2}$. The value in the bracket is obtained without the spin sum rule term, i.e., the upper limit for $J=0$, while the lower limit for $J=2$. The values $\Sigma_{\text {sum }}$ for DIAS ${ }^{(J=0)}(\mathrm{DGT})$ in Eq. (11) are calculated by using the same shell model wave functions as for $\Sigma \cdot \Sigma$ and shown in the sixth column in Table 1. The single particle values of $\Sigma_{\text {sum }}$ in Eq. (13) are given in the bracket in the sixth column. The value DIAS $S_{-}^{(J=0)}(\mathrm{DGT})$ with the single particle value of $\Sigma_{\text {sum }}$ is also listed in the fifth column with brackets. In all the nuclei in the present study, the value of $\Sigma_{\text {sum }}$ is one-fourth of $\hat{\Sigma} \cdot \hat{\Sigma}$ because of semi-closed configuration (either proton or neutron configuration is a closed shell).

The values $\Sigma_{\text {sum }}$, equivalently $\hat{\Sigma} \cdot \hat{\Sigma}$, are almost negligible for p-shell nuclei ${ }^{6} \mathrm{He}$ and ${ }^{8} \mathrm{He}$, because of good SU(4) spin-isospin symmetry in which the spin transitions are allowed only for spin and isospin flip transition between $(S, T)=(0,1) \rightarrow(1,0)$. This selection turns out to be the selection of transitions $\left(J^{\pi}, T\right)=\left(0^{+}, 1\right) \rightarrow\left(1^{+}, 0\right)$ in ${ }^{6} \mathrm{He}$ and $\left(J^{\pi}, T\right)=\left(0^{+}, 2\right) \rightarrow\left(1^{+}, 1\right)$ in ${ }^{8} \mathrm{He}$, corresponding to the charge exchange GT transitions. The shell model values $\Sigma_{\text {sum }}$ are smaller than the single-particle values in other nuclei by a factor $1.2 \sim 2.5$. The effect of $\hat{\Sigma} \cdot \hat{\Sigma}$ term for $J=0$ and $J=2$ sum rules are negligible for ${ }^{6} \mathrm{He}$ and ${ }^{8} \mathrm{He}$, while it amounts at most $20 \%$ in other nuclei with $T>1$ and it becomes much larger in $T=1$ nuclei such as ${ }^{14} \mathrm{C}$, ${ }^{18} \mathrm{O}$ and ${ }^{42} \mathrm{Ca}$.

In $N>Z$ nuclei, the sum rule values of DGT transitions are approximately proportional to $(2 \mathrm{~J}+1)$ factor, i.e., the value for $J=2$ is 5 times larger than that for $J=0$ in the same nucleus. However, this proportionality is strongly modified in $N \sim Z$ nuclei. In the extreme, the sum rule for $J=2$ transitions is smaller than that for $J=0$ in the nuclei ${ }^{6} \mathrm{He},{ }^{14} \mathrm{C}$, and ${ }^{18} \mathrm{O}$ with $N=Z+2$, and the two values are almost equal in ${ }^{42} \mathrm{Ca}$.

It is noticed that the DGT transition to DIAS state is much smaller than the sum rule values of DGT transitions for $J=0$ and $J=2$ states in nuclei with $N>Z+2$. However, in $T=1$ nuclei, the DGT transition to DIAS state is comparable with the DGT sum rules (5) and (8). This characteristics turns out the possibility to extract the unit cross section for DGT transition in $T=1$ nuclei, especially ${ }^{14} \mathrm{C}$ and ${ }^{18} \mathrm{O}$, since the DIAS state might have a narrow width and may be distinguished clearly from other DGT states in double charge exchange reactions [3]. This argument is entirely benefitted from the fact that the spinisospin response is much favorable that the isospin response in the medium energy charge exchange reactions with $E_{\text {lab }} \sim 200 \mathrm{MeV} / \mathrm{u}$.

\section{Summary}

In summary, we studied the sum rules of DGT excitations by the operator $\left(\sigma t_{-}\right)^{2}$. In nuclei $N>>Z$, the $J^{\pi}=2^{+}$excitations dominate the DGT strength, due to the multipole factor $(2 J+1)$, more than $0^{+}$excitations. However, in nuclei with $N \sim Z$, the $0^{+}$excitations become substantially strong, even larger than $2^{+}$excitations in light nuclei with $T=1,{ }^{14} \mathrm{C}$ an ${ }^{18} \mathrm{O}$. The excitation to DIAS is also studied through $\left(\sigma t_{-}\right)^{2}$ and $t_{-}^{2}$ operators to investigate the possibility to extract the unit cross sections for DGT strength. We pointed out that the strength of DIAS excitations by $\left(\sigma t_{-}\right)^{2}$ operator is competitive to DGT strength in the light $T=1$ nuclei. This characteristic feature may give a good opportunity to extract the unit cross section for DGT strength.

\section{Acknowledgments}

This work was supported in part by JSPS KAKENHI Grant Numbers JP19K03858.

\section{References}

[1] M. Takaki, T. Uesaka et al., Proposal for experiment at RCNP, "Search for double Gamow Teller giant resonances in ${ }^{48} \mathrm{Ti}$ via the heavy-ion double charge exchange ${ }^{48} \mathrm{Ca}\left({ }^{12} \mathrm{C},{ }^{12} \mathrm{Be}\left(0_{2}^{+}\right)\right)$reaction" (2015).

[2] F. Cappuzzello, C. Agodi, M. Bond? D. Carbone, M. Cavallaro, A. Foti, Journal of Physics: Conference Series 630, 012018 (2015).

[3] M. Kaletka, K. K. Seth, A. Saha, D. Barlow, and D. Kielczewska, Physics Letters B 199, 336 (1987).

[4] S. Mordechai and C.F. Moore, Nature 352, 393 (1991)

[5] H. Ward et al. , Phys. Rev. Lett. 70, 3209 (1993)

[6] Ph. Chomaz and N. Frascaria, Phys. Rep. 252, 275 (1995).

[7] J. Blomgren et al., Phys. Lett. B362, 34 (1995).

[8] T. Uesaka, M. Takaki et al., Proposal for Nuclear Physics Experiment at RI Beam Factory " Search for Double Gamow-Teller Giant Resonances in $\beta \beta$-decay nuclei via the heavy-ion double charge exchange ${ }^{48} \mathrm{Ca}\left({ }^{12} \mathrm{C},{ }^{12} \mathrm{Be}\left(\mathrm{O}_{2}^{+}\right)\right)$reaction" (2016).

[9] N. Shimizu, J. Menendez and K. Yako, Phys. Rev. Lett. 120, 142502 (2018).

[10] N. Auerbach and B. M. Loc, Phys. Rev. C98, 064301 (2018).

[11] P. Vogel, M. Ericson and J. D. Vergados, Phys. Lett. B212, 259(1988).

[12] D. C. Zheng, L. Zamick and N. Auerbach, Phys. Rev. C40, 936 (1989).

[13] K. Muto, Phys. Lett. B277, 13 (1992).

[14] H. Sagawa and T. Uesaka, Phys. Rev. C 94, 064325 (2016).

[15] X. Roca-Maza, H. Sagawa and G. Colò, arXiv:1907.06368(2019).

[16] M. Ichimura, H. Sakai, and T. Wakasa, Prog. in Part. Nucl. Phys. 56, 446 (2006).

[17] X. Roca-Maza, G. Colò, and H. Sagawa, Phys. Rev. C 86, 031306(R)(2012).

[18] S. H. Shen, G. Colò and X. Roca-Maza, Phys. Rev. C 99, 034322(2019).

[19] K. Yako et al., Phys. Rev. Lett. 103, 012503 (2009). 